\title{
Interacting Relativistic Boson Fields in the De Sitter Universe with Two Space-Time Dimensions
}

\author{
Rodolfo Figari`, Raphael Höegh-Krohn, and Chiara R. Nappi ${ }^{\star}$ \\ Institute of Mathematics, University of Oslo, Blindern Oslo 3, Norway
}

Received February 11, 1975

\begin{abstract}
The positive temperature Gibbs state of a scalar boson field with a relativistic local selfinteraction in two space-time dimensional Minkowski universe as constructed in [1] is not relativistic invariant. We prove in this paper that the corresponding state in the De Sitter universe is actually relativistic invariant if the temperature is given by $T=\frac{1}{2 \pi R}$ where $R$ is the constant radius of curvature of the De Sitter universe. Moreover the construction gives that the Schwinger functions or imaginary time Wightman functions are the moments of a generalized Markoff process on the sphere of radius $R$.
\end{abstract}

\section{Introduction}

In a recent paper [1], a Markoff field approach has been applied to the study of statistical mechanics for interacting bosons in two space-time dimensions, without cutoff. The Gibbs state at positive temperature is explicitely constructed and it is proved that for every positive temperature the thermodynamic limit exists and is unique for the polynomial and exponential interactions. This is achieved by showing that the Schwinger functions at positive temperature $\frac{1}{\beta}$ (the imaginary time Gibbs states at temperature $\frac{1}{\beta}$ ) are equal to the Schwinger functions (in the usual terminology) for the interacting field in a periodic box of length $\beta$. In turn this is proved expressing the functions $S^{\beta}$ by means of a F.K.N.type formula which links the Gibbs state at temperature $\frac{1}{\beta}$ to a Markoff field on the cylinder $S_{\beta} \times \mathbb{R}\left(S_{\beta}\right.$ is the circle of length $\beta$ ) which becomes the euclidean Markoff field on $\mathbb{R}^{2}$ associated with the 0 temperature theory when the curvature of the cylinder goes to 0 , i.e. when $\beta \rightarrow \infty$.

The lack of relativistic covariance contained in the definition of Gibbs state has, as an imaginary time counter-part, the lack of euclidean covariance of the associated Markoff field and, roughly speaking, is measured by the magnitude of $\frac{1}{\beta}$ or, in other words, by the radius of curvature in time direction of the manifold supporting the associated Markoff field. This suggests the possibility of carrying out the construction of an invariant Gibbs state for a boson scalar field in a finite, homogeneous, isotropic 2-dimensional universe with constant curvature in

\footnotetext{
* Supported in part by the Italian Ministry of Education.
} 
spatial direction (the so called De Sitter universe) by means of a Markoff field on a sphere, at least if the temperature of this universe is chosen equal to $(2 \pi R)^{-1}$, where $R$ is the radius of curvature of the universe.

The symmetry of the structure of the De Sitter universe makes it easier to deal with the difficulties arising from the lack of a global system of coordinates. On the other hand, the De Sitter Universe presents the advantage of having finite spatial extension and hence no infinite volume problems.

In this paper we develop this idea merely by adapting the results set up in [1] to this case, showing that there exists the positive temperature state or the Gibbs state at temperature $\frac{1}{\beta}$, for $\beta=2 \pi R$, for a boson field in two-dimensional spacetime with polynomial and exponential interaction and without cutoff and that this state is invariant under the De Sitter group.

In this connection, the new results [2] on the removal of the momentum cutoff for polynomial interactions in 3 space-time dimensions in the flat case suggest that, hopefully, in a De Sitter universe, because of its finite spatial extension, it will be possible to get the invariant Gibbs state in space-time dimensions higher than two.

\section{The Two Dimensional De Sitter Universe}

The metric of the 2-dimensional De Sitter universe is

$-d s^{2}=\phi^{2}\left(x^{1}, x^{0}\right)\left[\left(d x^{1}\right)^{2}-\left(d x^{0}\right)^{2}\right]$

with

$$
\phi\left(x^{1}, x^{0}\right)=\left(1+\frac{\left(x^{1}\right)^{2}-\left(x^{0}\right)^{2}}{4 R^{2}}\right)^{-1} \quad R>0
$$

corresponding to a static, homogeneous and isotropic solution of the Einstein field equations, with the condition

$$
\mu^{0}+p^{0}=0 \quad(c=1),
$$

where $\mu^{0}$ is the density and $p^{0}$ the pressure of the universe [3]. The two dimensional De Sitter universe can be embedded in a three dimensional flat space. It corresponds there to the hyperboloid:

$$
\xi_{1}^{2}+\xi_{2}^{2}-\xi_{3}^{2}=R^{2}
$$

with

$$
\begin{aligned}
& \xi_{1}=\phi\left(x^{1}, x^{0}\right) x^{1} \\
& \xi_{2}=R \phi\left(x^{1}, x^{0}\right)\left(1-\frac{\left(x^{1}\right)^{2}-\left(x^{0}\right)^{2}}{4 R^{2}}\right) \\
& \xi_{3}=\phi\left(x^{1}, x^{0}\right) x^{0}
\end{aligned}
$$

and the metric:

$$
-d s^{2}=d \xi_{1}^{2}+d \xi_{2}^{2}-d \xi_{3}^{2} .
$$


In turn by means of the $\xi$ 's it is possible to define the Lemaitre-Robertson coordinates $x^{\prime}, t^{\prime}$ which make the De Sitter universe's geometry more transparent:

$$
\begin{aligned}
& \xi_{1}=x^{\prime} e^{\frac{t^{\prime}}{R}} \quad \xi_{2}=R\left(\cosh \frac{t^{\prime}}{R}-\frac{x^{\prime 2}}{2 R^{2}} e^{\frac{t^{\prime}}{R}}\right) \\
& \xi_{3}=R\left(\sinh \frac{t^{\prime}}{R}+\frac{x^{\prime 2}}{2 R^{2}} e^{\frac{t^{\prime}}{R}}\right)
\end{aligned}
$$

and

$$
-d s^{2}=e^{\frac{2 t^{\prime}}{R}} d x^{\prime 2}-d t^{\prime 2} .
$$

In this system of coordinates the scalar and vector potential of the gravitational fields are 0 . The trajectories of light rays are straight lines. Furthermore, for every fixed time $t$, the metric is euclidean and the spatial distance from the origin of a point of coordinate $x^{\prime}$ is:

$$
l=e^{\frac{t^{\prime}}{R}} x^{\prime} .
$$

It is simple to verify that, because of this "expansion" the furthest place from where an observer placed in the origin of the spatial coordinates $\left(x^{\prime}=0\right)$ can "have informations" (by means of light signals) is at distance which, for every $t^{\prime}$, is equal to the constant $R$, the so called horizon of the universe. From (1.5) it follows that the visible part of the universe consists, for this observer, of the points that at time $t^{\prime}$ have coordinates $x^{\prime}$ whose absolute value does not exceed $e^{\frac{-t^{\prime}}{R}} R$.

The De Sitter group is the group of transformations of the hyperboloid (1.1) onto itself, which leave invariant the metric (1.2); in other words it is the group of the orthogonal transformations in the euclidean space of the coordinates $\xi_{1}, \xi_{2}$, and $\xi_{3}^{\prime}=i \xi_{3}$. The induced action of a transformation $\xi_{i} \rightarrow \hat{\xi}_{i}$ of the De Sitter group on the coordinates $x^{\prime} ; t^{\prime}$ :

$$
x^{\prime}=x^{\prime}\left(\xi_{i}\right) \rightarrow \hat{x}^{\prime}=x^{\prime}\left(\hat{\xi}_{i}\right)
$$

leaves (1.4) form invariant, i.e. $g_{i k}\left(x^{\prime}, t^{\prime}\right)=g_{i k}\left(\hat{x}^{\prime}, \hat{t}^{\prime}\right)$. This is obviously true for any pair of generalized coordinates describing the De Sitter universe and in this sense the transformations of the De Sitter group are said to link "equivalent systems".

In the coordinates $\xi_{1}, \xi_{2}, \xi_{3}^{\prime}$ the generators of the De Sitter group are the following well known generators

$$
\begin{aligned}
& J_{1,2}=\xi_{1} \frac{\partial}{\partial \xi_{2}}-\xi_{2} \frac{\partial}{\partial \xi_{1}}, \quad J_{2,3}=\xi_{2} \frac{\partial}{\partial \xi_{3}^{\prime}}-\xi_{3}^{\prime} \frac{\partial}{\partial \xi_{2}}, \\
& J_{3,2}=\xi_{3}^{\prime} \frac{\partial}{\partial \xi_{2}}-\xi_{2} \frac{\partial}{\partial \xi_{3}^{\prime}} .
\end{aligned}
$$

The one parameter subgroup of time translations should be of course a non compact one dimensional subgroup of the De Sitter group. It is well known that there is only one such subgroup up to inner automorphisms, and its generator 
is in fact $J_{3,2}$. We therefore introduce the coordinates $\varphi, t$ by

$$
\begin{aligned}
& \xi_{1}= R \sin \frac{\varphi}{R}, \quad \xi_{2}=R \cosh \frac{t}{R} \cos \frac{\varphi}{R}, \quad \xi_{3}=R \sinh \frac{t}{R} \cos \frac{\varphi}{R} \\
&-\infty \leqq t \leqq \infty, \quad-\frac{\pi R}{2} \leqq \varphi \leqq \frac{\pi R}{2}
\end{aligned}
$$

to get the points in the De Sitter universe parametrized in such a way that the transformation induced in the De Sitter universe by the translation $t \rightarrow t+s$ in the coordinates (1.7) is the action of the one parametric time subgroup of the De Sitter group.

Actually these new coordinates do not cover the whole De Sitter universe, but only the portion

$$
-R \leqq \xi_{1} \leqq R, \xi_{2} \geqq 0 \quad-\infty \leqq \xi_{3} \leqq \infty .
$$

However, they cover exactly the visible universe of an observer who is at $x^{\prime}=0$ when $t^{\prime}=0$ (or equivalently $\varphi=0$ and $t=0$ ). In fact we have already mentioned that the visible world of this observer at "time" $t^{\prime}$ is $\left\{x^{\prime}|| x^{\prime} \mid \leqq R e^{\left.-\frac{t^{\prime}}{R}\right\}}\right.$, which just by (1.3) implies (1.8). In the $(\varphi, t)$ coordinates the metric reads

$$
-d s=d \varphi^{2}-\cos ^{2} \frac{\varphi}{R} d t^{2} \text {. }
$$

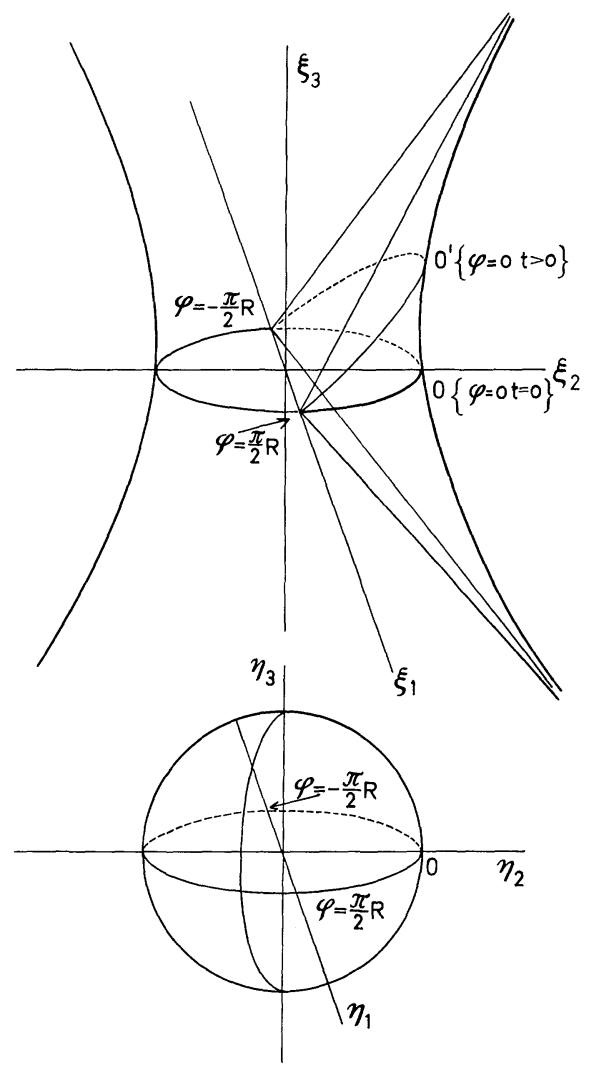


The first drawing represents the visible world of an observer in the origin 0 .

$$
\begin{aligned}
& \xi_{1}= R \sin \frac{\varphi}{R} \quad \xi_{2}=R \cosh \frac{t}{R} \cos \frac{\varphi}{R} \quad \xi_{3}=R \sinh \frac{t}{R} \cos \frac{\varphi}{R} \\
&-\infty \leqq t \leqq \infty-\frac{\pi}{2} R \leqq \varphi \leqq \frac{\pi}{2} R .
\end{aligned}
$$

The transformation $t \rightarrow-i t$ maps the imaginary sphere $\xi_{1}, \xi_{2}, \xi_{3}^{\prime}=i \xi_{3}$ onto the sphere

$$
\begin{gathered}
\eta_{1}=R \sin \frac{\varphi}{R} \quad \eta_{2}=R \cos \frac{t}{R} \cos \frac{\varphi}{R} \quad \eta_{3}=R \sin \frac{t}{R} \cos \frac{\varphi}{R} \\
0 \leqq t \leqq 2 \pi \quad-\frac{\pi}{2} R \leqq \varphi \leqq \frac{\pi}{2} R .
\end{gathered}
$$

\section{The Hamiltonian of the De Sitter Scalar Field}

Formally we can construct the invariant classical action for the classical field $\phi$

$$
A=\int_{-\infty}^{\infty} \int_{-\frac{\pi}{2} R}^{\frac{\pi}{2} R} L\left(\phi, \frac{\partial \phi}{\partial \varphi}, \frac{\partial \phi}{\partial t}\right) \sqrt{|g|} d \varphi d t
$$

where $L$ is a scalar lagrangian density and $|g|$ is the determinant of the metric matrix. Let us choose the scalar field lagrangian

$$
L=\frac{1}{2}\left(g^{i k} \frac{\partial}{\partial x^{i}} \phi \frac{\partial}{\partial x^{k}} \phi-m^{2} \phi^{2}\right)
$$

and the metric (1.9) so that

$$
\begin{aligned}
A & =\frac{1}{2} \int_{-\infty}^{\infty} \int_{-\frac{\pi}{2} R}^{\frac{\pi}{2} R}\left[\left(\cos ^{2} \frac{\varphi}{R}\right)^{-1}\left(\frac{\partial \phi}{\partial t}\right)^{2}-\left(\frac{\partial \phi}{\partial \varphi}\right)^{2}-m^{2} \phi^{2}\right] \cos \frac{\varphi}{R} d \varphi d t \\
& =\frac{1}{2} \int_{-\infty}^{\infty} \int_{-\frac{\pi}{2} R}^{\frac{\pi}{2} R}\left[\left(\frac{\partial \phi}{\partial t}\right)^{2}-\cos ^{2} \frac{\varphi}{R}\left(\frac{\partial \phi}{\partial \varphi}\right)^{2}-m^{2} \cos ^{2} \frac{\varphi}{R} \phi^{2}\right]\left(\cos \frac{\varphi}{R}\right)^{-1} d \varphi d t .
\end{aligned}
$$

The invariance with respect to the translations of $t$ provides us with the conserved quantity

$$
H=\frac{1}{2} \int_{-\frac{\pi}{2} R}^{\frac{\pi}{2} R}\left[\left(\frac{\partial \phi}{\partial t}\right)^{2}+\cos ^{2} \frac{\varphi}{R}\left(\frac{\partial \phi}{\partial \varphi}\right)^{2}+m^{2} \cos ^{2} \frac{\varphi}{R} \phi^{2}\right]\left(\cos \frac{\varphi}{R}\right)^{-1} d \varphi .
$$

For every arbitrary closed interval $I^{\prime}=\left[a^{\prime}, b^{\prime}\right]$ strictly contained in $\left[-\frac{\pi}{2} R, \frac{\pi}{2} R\right]$ the form (2.0) can be written

$$
H_{I}=\frac{1}{2}(\dot{\phi}, \dot{\phi})+\frac{1}{2}\left(\phi, A_{I^{\prime}}^{2} \phi\right)
$$

where $($,$) is the scalar product in L^{2}\left(I^{\prime},\left(\cos \frac{\varphi}{R}\right)^{-1} d \varphi\right)$ and $A_{I^{\prime}}^{2}$ is the unique strictly positive operator with fixed selfadjoint boundary conditions defined, via the representation theorem, by the bilinear form

$$
t(u, v)=\int_{I^{\prime}}\left[\cos ^{2} \frac{\varphi}{R} \frac{d u}{d \varphi} \frac{d u}{d \varphi}+m^{2} \cos ^{2} \frac{\varphi}{R} u v\right]\left(\cos \frac{\varphi}{R}\right)^{-1} d \varphi .
$$


The (2.1) can be put in more suitable form by means of the unitary transformation $U$

$$
L^{2}\left(I^{\prime},\left(\cos \frac{\varphi}{R}\right)^{-1} d \varphi\right) \stackrel{U}{\longrightarrow} L^{2}(I, d x)
$$

given by

$$
(U v)(x)=v^{\prime}(x)=v\left(2 \operatorname{Rarctg} e^{\frac{x}{R}}-\frac{\pi}{2} R\right),
$$

where $I=[a, b]$ with $a=R \log \operatorname{tg}\left(\frac{\pi}{4}+\frac{a^{\prime}}{2 R}\right)$ and $b=R \log \operatorname{tg}\left(\frac{\pi}{4}+\frac{b^{\prime}}{2 R}\right) \cdot$ On $L^{2}(I, d x)$ we get from the form (2.1)

$$
t^{\prime}\left(u^{\prime}, v^{\prime}\right)=\int_{I}\left[\frac{d u^{\prime}}{d x} \frac{d v^{\prime}}{d x}+\left(\cosh \frac{x}{R}\right)^{-2} u^{\prime} v^{\prime}\right] d x
$$

The following properties are well known and easily checked for any such form.

1) The unique strictly positive selfadjoint operator $A_{I}$ associated with (2.3) has discrete spectrum with eigenvalues $\lambda_{1}{ }^{2}<\lambda_{2}{ }^{2} \ldots<\lambda_{n}{ }^{2}<\ldots$ of finite multiplicity and $\lim _{n} \lambda_{n}=\infty$ with the same degree of increase as $n^{2}$.

Moreover $e^{-s A_{I}^{2}}, s>0$ is of trace class. (These are simple consequences of the fact that on $L^{2}(I, d x)$

$$
-\Delta_{I}+m^{2} \geqq A_{I}^{2} \geqq-\Delta_{I}+c
$$

for some $c>0$ and of the minimax principle [4].)

2) The square root $A_{I}$ of $A_{I}^{2}$ is selfadjoint, strictly positive, with discrete spectrum consisting of eigenvalues of finite multiplicity which grow to infinity at least as $n$ does; $e^{-s A_{I}}, s>0$ is of trace class. Furthermore $D\left(A_{I}\right)=D\left(t^{\prime}\right)$ and $D\left(A_{I}^{2}\right)$ is a core of $A_{I}$. To achieve the non formal Hamiltonian of the quantized theory, let us consider the Hilbert space $h_{I}$ of the real functions of $\varphi$ belonging to $D\left(A_{I}\right)=$ $D\left(t^{\prime}\right)$ equipped by the norm:

$$
\|f\|^{2}=2\left(f, A_{I} f\right) \equiv\langle f, f\rangle
$$

where (,) is the internal product on $L^{2}(I, d x)$. Let $L^{2}\left(d \mu_{h_{I}}\right)$ be the space of the complex functions square integrable w.r.t. the normal distribution on $h_{I} . L^{2}\left(d \mu_{h_{I}}\right)$ is isomorphic with the symmetric tensor algebra on $h_{I}$, i.e. the Fock space $\mathscr{F}\left(h_{I}\right)$ constructed on $h_{I}[5]$.

We define $H_{I}{ }^{0}$ as the selfadjoint generator of the strongly continuous group of unitary operators $\Gamma\left(e^{i t A_{I}}\right)$ (the second quantization of $\left.e^{i t A_{I}}\right)$ on $L^{2}\left(d \mu_{h_{I}}\right)$.

The property 2) stated above implies immediately that $e^{-s H_{I}{ }^{0}}$ is of trace class and that

$$
\operatorname{tr} e^{-s H_{I}{ }^{0}}=\left|1-e^{-s A_{I}}\right|^{-1}=e^{-\operatorname{tr} \log \left(1-e^{\left.-s A_{I}\right)}\right.} .
$$

\section{The Gibbs State for the Free Scalar Quantum Field}

3.1. We can now define the Gibbs state at positive temperature $T=\frac{1}{\beta}$ with $\beta=2 \pi R$ for the free De Sitter scalar field of mass $m$ by

$$
\omega_{I}^{0}(B)=\left(\operatorname{tr} e^{-2 \pi R H_{I}{ }^{0}}\right)^{-1} \operatorname{tr}\left(B e^{-2 \pi R H_{I}{ }^{0}}\right),
$$


where $B \in B\left(L^{2}\left(d \mu_{h_{I}}\right)\right)$, the $C^{*}$-algebra of bounded operators on $L^{2}\left(d \mu_{h_{I}}\right)$. To construct it explicitly we have to state a lemma, proved in [1], which will provide us with the main technical tool in the following. We need first to introduce some notations.

Let $Q_{I}$ be the Hilbert space of the functions $\xi^{\prime}(\varphi, t)$ with $\varphi \in I^{\prime}=\left[a^{\prime}, b^{\prime}\right]$ and $t \in[0,2 \pi R]$ which are periodic in $t$, satisfy for every $t$ the boundary condition of $A_{I^{\prime}}^{2}$ and have the norm:

finite.

$$
\left|\xi^{\prime}, \xi^{\prime}\right|=\int_{0}^{2 \pi R} \int_{I^{\prime}}\left[\left(\cos ^{2} \frac{\varphi}{R}\right)^{-1}\left(\frac{\partial \xi^{\prime}}{\partial t}\right)^{2}+\left(\frac{\partial \xi^{\prime}}{\partial \varphi}\right)^{2}+m^{2} \xi^{\prime 2}\right] \cos \frac{\varphi}{R} d \varphi d t
$$

Equivalent characterization of $Q_{I}$ are:

1) the Hilbert space of the functions $\xi(x, t), x \in I=[a, b] t \in[0,2 \pi R]$ which are periodic in $t$, satisfy for every $t$ the boundary conditions on $A_{I}^{2}$ and have the norm

$$
|\xi, \xi|=\int_{0}^{2 \pi R} \int_{I}\left[\left(\frac{\partial \xi}{\partial t}\right)^{2}+\left(\frac{\partial \xi}{\partial x}\right)^{2}+\left(\cosh \frac{x}{R}\right)^{-2} m^{2} \xi^{2}\right] d x d t
$$

finite;

2) the Hilbert space of the functions $\eta(t)$ from the circle of length $2 \pi R$ in $L^{2}\left(I^{\prime},\left(\cos \frac{\varphi}{R}\right)^{-1} d \varphi\right)$ equipped by the norm:

$$
\left\||\eta \||=\int_{0}^{2 \pi R}\left[\left(\frac{d \eta}{d t}, \frac{d \eta}{d t}\right)+\left(\eta, A_{I}^{2}, \eta\right)\right] d t\right.
$$

where $($,$) is the inner product in L^{2}\left(I^{\prime},\left(\cos \frac{\varphi}{R}\right)^{-1} d \varphi\right) \cdot Q_{I}$ will indicate one of the above representations equivalently. Let $d \mu_{Q_{I}}$ denote the normal distributions over $Q_{I}$ and $E_{I}{ }^{0}(\cdot)=\int \cdot d \mu_{Q_{I}}$ be the expectation w.r.t. $d \mu_{Q_{r}} . E_{I}{ }^{0}$ can be as well characterized as the expectation w.r.t. the generalized gaussian process $\Phi^{\prime}$ on $[0,2 \pi R] \times I^{\prime}$ with mean 0 and covariance given by the Green function $G_{I^{\prime}}\left(\varphi, \varphi^{\prime}\right.$; $\left.t-t^{\prime}\right)$ of the selfadjoint operator associated with the form (3.1) on the space of the functions on the sphere cut along the "parallels" $\varphi=a^{\prime}$ and $\varphi=b^{\prime}$, square integrable w.r.t. the measure $d \omega=\cos \frac{\varphi}{R} d \varphi d t$. Moreover $E_{I}{ }^{0}$ can be characterized as the expectation w.r.t. the generalized gaussian process $\Phi$ on $[0,2 \pi R] \times I$ with mean 0 and covariance given by the Green function $G_{I}\left(x, x^{\prime} ; t-t^{\prime}\right)$ for the selfadjoint operator

$$
-\frac{\partial^{2}}{\partial t^{2}}-\frac{\partial^{2}}{\partial x^{2}}+\left(\cosh \frac{x}{R}\right)^{-2} m^{2}=-\frac{\partial^{2}}{\partial t^{2}}+A_{I}^{2}
$$

with the corresponding selfadjoint boundary condition. With respect to the variable $t$, it is then an ordinary homogeneous process and in particular the sharptime stochastic variables $\Phi(f, t)$ are well defined for $t \in[0,2 \pi R]$ and $f \in C_{0}^{\infty}(\operatorname{Int} I)$ by the covariance

$$
E_{I}{ }^{0}(\Phi(f, 0) \Phi(g, t))=\left(f,\left(2 A_{I}\left(1-e^{-2 \pi R A_{I}}\right)\right)^{-1}\left(e^{-t A_{I}}+e^{-(2 \pi R-t) A_{I}}\right) g\right),
$$

where $($,$) is the scalar product in L^{2}(I, d x)$. 
Finally we note that the unitary transformation $\xi^{\prime}(\varphi, t) \rightarrow \xi^{\prime}(\varphi, t+\tau)$ on $Q_{I}$ induces a $d \mu_{Q_{I}}$-measure-preserving transformation and hence an isometric transformation on every $L^{P}\left(d \mu_{Q_{I}}\right), 1 \leqq p \leqq \infty$, that will be indicated by $O_{\tau}$.

With these definitions we have:

Lemma 3.0. If $F_{0}, F_{1}, \ldots, F_{m-1}$ are in $L^{\infty}\left(d \mu_{h_{I}}\right)$ and $0=s_{0} \leqq s_{1} \ldots \leqq s_{m}=2 \pi R$ then

$\operatorname{tr}\left(F_{0} e^{-s_{1} H_{I}{ }^{0}} F_{1} e^{-\left(s_{2}-s_{1}\right) H_{I}{ }^{0}} \ldots F_{m-1} e^{-\left(2 \pi R-s_{m-1}\right) H_{I}{ }^{0}}\right)$

$=\left|1-e^{-2 \pi R A_{I}}\right|^{-1} E_{I}^{0}\left[\prod_{i=0}^{m-1} O_{s_{i}} F_{i}\right]$.

Remark. The lemma remains true if, instead of $F_{i}$ 's in $L^{\infty}\left(d \mu_{h_{\mathrm{I}}}\right)$ we put in the left hand side of (3.3) the time zero relativistic fields $\phi\left(f_{i}\right), f_{i} \in C_{0}^{\infty}(\operatorname{Int} I)$, i.e. the linear functionals on $h_{I}$ space given by $[\phi(f)](\eta)=(\eta, f)$ where $($, $)$ is the scalar product in $L_{2}(I, d x)$.

In fact, in this case, in the right hand side of (3.3) will appear just the $m$-th momentum of the sharp time stochastic variables $\Phi\left(f_{i}, s_{i}\right)$.

3.2. We define now the $C^{*}$-automorphism on $B\left(L^{2}\left(d \mu_{h_{I}}\right)\right)$

$\alpha_{I}^{0}[t](B)=e^{-i t H_{I}{ }^{0}} B e^{i t H_{I}{ }^{0}}$.

For $B$ and $C$ in $B\left(L^{2}\left(d \mu_{h_{I}}\right)\right)$ the Gibbs state has the following properties:

a) $\omega_{I}^{0}\left(B \cdot \alpha_{I}^{0}[t](C)\right)=\omega_{I}^{0}\left(\alpha_{I}^{0}[-t](B) \cdot C\right)$.

b) (3.4) is analytic in $t$ in the strip $-2 \pi R<\operatorname{Im} t<0$, continuous and uniformly bounded in the closure of the strip.

c) (3.4) satisfies the KMS condition on the boundary

$\omega_{I}^{0}\left(B \cdot \alpha_{I}^{0}[t-i 2 \pi R](C)\right)=\omega_{I}^{0}\left(C \cdot \alpha_{I}^{0}[-t](B)\right)$.

d) If $F_{0} \ldots F_{m} \in L^{\infty}\left(d \mu_{h_{I}}\right)$ then $\omega_{I}^{0}\left(F_{0} \alpha_{I}^{0}\left[t_{1}\right]\left(F_{1}\right) \ldots \alpha_{I}^{0}\left[t_{m}\right]\left(F_{m}\right)\right)$ is analytic in $-2 \pi R<\operatorname{Im} t_{m}<\ldots \operatorname{Im} t_{1}<0$, continuous and uniformly bounded in the closure of the hyperstrip and its value at imaginary points $t_{k}=-i s_{k}, s_{k} \in \mathbb{R}$ and $0=s_{0} \leqq$ $s_{1} \ldots \leqq s_{m+1}=2 \pi R$, is given by

$$
\omega_{I}^{0}\left(F_{0} \alpha_{I}^{0}\left[-i s_{1}\right]\left(F_{1}\right) \ldots \alpha_{I}^{0}\left[-i s_{m}\right]\left(F_{m}\right)\right)=E_{I}^{0}\left(\prod_{k=0}^{m} O_{s_{k}} F_{k}\right) .
$$

As in the remark of the Lemma 3.0, the property d) extends to the case in which $F_{0} \ldots F_{m}$ are substituted by the fields $\phi\left(f_{0}\right) \ldots \phi\left(f_{m}\right)$. We will call the quantities

$$
W_{I}^{0}\left(f_{0}, O \ldots f_{m}, t_{m}\right)=\omega_{I}^{0}\left(\phi\left(f_{0}\right) \alpha_{I}^{0}\left[t_{1}\right] \phi\left(f_{1}\right) \ldots \alpha_{I} o\left[t_{m}\right] \phi\left(f_{m}\right)\right)
$$

the Wightman functions for the free De Sitter scalar field in the volume I. The corresponding imaginary time quantities will be called Schwinger functions for the same field.

Let now $\Omega$ be an open interval $\Omega \subset I$ and $V(f), U(f), f \in C_{0}^{\infty}(\Omega)$ the standard unitary representation of the commutation relations in Weil form on $L^{2}\left(d \mu_{h_{\mathrm{I}}}\right)$. Explicitly, $V(f)$ can be taken to be the operator of multiplication by $e^{i(\psi, f)}$ for $\psi \in h_{I}$ and $U(g)$ defined by $U(g) F(\psi)=F(\psi+g)$ for every $F \in L^{2}\left(d \mu_{h_{I}}\right)$. Let us introduce the definitions:

$\mathscr{A}_{0}(\Omega)$ is the smallest norm closed algebra in $B\left(L^{2}\left(d \mu_{h_{I}}\right)\right)$ which contains $V(g)$ and $U(g), g \in C_{0}^{\infty}(\Omega)$. 
$\overline{\mathscr{A}}_{0}(\Omega)$ is its strong closure in $B\left(L^{2}\left(d \mu_{h_{L}}\right)\right)$.

$\overline{\mathscr{A}}_{0}$ denotes the norm closure of $\bigcup_{\Omega}\left[\mathscr{A}_{0}(\Omega) \mid \Omega \subset \mathbb{R}\right]$.

As in the flat case the two following properties hold:

1) $\overline{\mathscr{A}}_{0}(\Omega)$ does not depend on the choice of the interval $I$ as far as $\Omega \subset I$.

Because $\mathscr{A}_{0}(\Omega)$ is a norm closed algebra faithfully represented in every $B\left(L^{2}\left(d \mu_{h_{I}}\right)\right)$, it does not depend on the interval $I$, for $\Omega \subset I$. To see that $\overline{\mathscr{A}}_{0}(\Omega)$, too, does not depend on $I$, we have to show that if $\Omega \subset I_{1} \subset I_{2}$ the strong topologies in $B\left(L^{2}\left(d \mu_{h_{I_{1}}}\right)\right)$ and in $B\left(L^{2}\left(d \mu_{h_{I_{2}}}\right)\right)$ induce equivalent topologies on $\mathscr{A}_{0}(\Omega)$. In turn it is enough to prove that the measures obtained conditionating the generalized gaussian process of mean 0 and covariance given by the Green functions of the operators $A_{I_{1}}$ and $A_{I_{2}}$ with respect to the $\sigma$-algebra generated by $\phi(f)$, with $f \in C_{0}^{\infty}(\Omega)$ are equivalent. This is so because in the representation (3.2) we have

$$
A_{I}^{2}=\frac{-\partial^{2}}{\partial x^{2}}+m^{2}\left(\cosh \frac{x}{R}\right)^{-2}
$$

as the selfadjoint operator on $L^{2}(I, d x)$ with some selfadjoint boundary condition at the endpoints of $I$. So we see that our free Hamiltonian is the same as the flat Hamiltonian with a quadratic perturbation. Since $\left(\cosh \frac{x}{R}\right)^{-2}$ is bounded below by a positive constant on the finite interval $I$, we get the equivalence from the corresponding equivalence in the flat case.

2) We have also the classical result about the finite propagation speed, i.e. if $B \in \overline{\mathscr{A}}_{0}(\Omega)$ then $\alpha_{I}^{0}[t](B) \in \overline{\mathscr{A}}_{0}\left(\Omega_{t}\right)$ where $\Omega_{t}=\{x \mid \operatorname{dist}(x, \Omega)<t\}$.

In fact the proof [6] in the flat case is based only on the abstract Fock structure and on the property of the fundamental solution of Klein-Gordon equation to propagate inside the light cone, that is a general property of any hyperbolic equation and applies as well in our case where the K.G. equation for the field is substituted by

$$
\frac{\partial^{2}}{\partial t^{2}} \phi(x, t)=-\left(-\frac{\partial^{2}}{\partial x^{2}}+m^{2}\left(\cosh \frac{x}{R}\right)^{-2}\right) \phi(x, t) .
$$

So $\alpha_{I}^{0}[t]$ is independent of $I$ as far as $\Omega_{t} \subset I$. Let us denote this independent value by $\alpha_{t}{ }^{0}(B) . \alpha_{t}{ }^{0}(B)$ is then a $C^{*}$-isomorphism from $\overline{\mathscr{A}}_{0}(\Omega)$ into $\overline{\mathscr{A}}_{0}\left(\Omega_{t}\right)$ for any $\Omega$, hence it extends to a $C^{*}$-automorphism of $\overline{\mathscr{A}}_{0}$. We define now the local algebra $\mathscr{A}_{0}$ for the free field. Let $\mathscr{F}(\Omega)$ be the class of functions in $L^{\infty}\left(d \mu_{h_{I}}\right)$ that are bounded continuous functions of a finite number of fields $\phi\left(f_{1}\right) \ldots \phi\left(f_{n}\right)$ with $f_{1} \ldots f_{n} \in C_{0}^{\infty}(\Omega)$. $\mathscr{A}_{0}$ will be the smallest norm closed $C^{*}$-algebra in $\mathscr{A}_{0}$ containing all the operators of the form $\alpha_{t}^{0}(F), \forall F \in \mathscr{F}(\Omega)$. $\mathscr{A}_{0}$ is obviously invariant under $\alpha_{t}{ }^{0}$.

3.3. We are now in condition to state the main theorem on the existence of the limit state $\omega^{0}$. Moreover we want to show that the infinite volume limit Wightman functions have covariance property with respect to the De Sitter group.

Theorem 3.1. If $\mathscr{A}_{0}$ is the local algebra for the free field and $\alpha_{t}^{0}$ is the $C^{*}$ automorphism defined above, then on $\mathscr{A}_{0}$ there exists a state $\omega^{0}$ with the following properties:

i) $\omega^{0}\left(B \cdot \alpha_{t}^{0}(C)\right)=\omega^{0}\left(\alpha_{-t}^{0}(B) \cdot C\right)$. 
ii) $\omega^{0}\left(B \cdot \alpha_{t}^{0}(C)\right)$ is analytic in the strip $-2 \pi R<\operatorname{Im} t<0$ and uniformly bounded in $-2 \pi R \leqq \operatorname{Im} t \leqq 0$ and satisfies the $\mathrm{KMS}$ condition on the boundary

$\omega^{0}\left(B \cdot \alpha_{t-i 2 \pi R}^{0}(C)\right)=\omega^{0}\left(C \cdot \alpha_{-t}^{0}(B)\right)$.

Furthermore if $F_{0} \ldots F_{n} \in \mathscr{F}(\Omega)$ then $\omega^{0}\left(F_{0} \alpha_{t_{1}}^{0}\left(F_{1}\right) \ldots \alpha_{t_{n}}^{0}\left(F_{n}\right)\right)$ is analytic in $0>\operatorname{Im} t_{1}>$ $\ldots>\operatorname{Im} t_{n}>-2 \pi R$, continuous and uniformly bounded in the closure of this hyperstrip and at imaginary time $t_{k}=-i s_{k}, 0=s_{0} \leqq s_{1} \ldots \leqq s_{n+1}=2 \pi R$ its value is given by

$$
\omega^{0}\left(F_{0} \alpha_{-i s_{1}}^{0}\left(F_{1}\right) \ldots \alpha_{-i s_{n}}^{0}\left(F_{n}\right)\right)=E^{0}\left(\prod_{k=0}^{n} O_{s_{k}} F_{k}\right) .
$$

Here $E^{0}$ is the expectation with respect to the generalized gaussian process with mean 0 and covariance given by the Green function $G\left(\varphi, \varphi^{\prime} ; t-t^{\prime}\right)$ for the operator $-\Delta_{s}+m^{2}$, where $-\Delta_{s}$ is the invariant laplacian on $L^{2}(d \omega)$ the square integrable functions on the sphere of radius $R$ w.r.t. the invariant measure $d \omega=\cos \frac{\varphi}{R} d \varphi d t$.

iii) if $B \in \mathscr{A}_{0}$ is in $\overline{\mathscr{A}}_{0}(\Omega)$ for some open bounded interval $\Omega$, then

$\omega^{0}(B)=\lim _{I \rightarrow \mathbb{R}} \omega_{I}{ }^{0}(B)$.

iv) if $f_{0}, f_{1}, \ldots, f_{n} \in C_{0}^{\infty}(\Omega)$ for some open bounded interval $\Omega$ then the limit

$$
W^{0}\left(f_{0}, 0, f_{1}, t_{1} \ldots f_{n}, t_{n}\right)=\lim _{I \rightarrow \mathbb{R}} \omega_{I}^{0}\left(\phi\left(f_{0}\right) \alpha_{t_{1}}^{0} \phi\left(f_{1}\right) \ldots \alpha_{t_{n}}^{0} \phi\left(f_{n}\right)\right)
$$

exists and is invariant under the De Sitter group.

The proof of i), ii), and iii) is contained in [1]. It follows from the finite propagation speed property, from Lemma 3.0 and from the convergence of the Green function of the operator associated with the form (3.1) on the cut sphere, with some selfadjoint boundary condition on $a^{\prime}$ and $b^{\prime}$, to the Green function of the operator $-\Delta_{s}+m^{2}$ where $-\Delta_{s}$ is the invariant laplacian on the whole sphere when $I^{\prime} \rightarrow\left[-\frac{\pi}{2} R, \frac{\pi}{2} R\right]$. The proof of the invariance of the Wightman functions requires only a slight modification of the Nelson's proof [6]. We already know that the limit (3.7) exists and is analytic in $0>\operatorname{Im} t_{1}>\ldots \operatorname{Im} t_{n}>-2 \pi R$.

Let $S^{0}\left(f_{0}, s_{0} ; f, s, \ldots t_{n}, s_{n}\right)=W^{0}\left(f_{0},-i s_{0} ; f_{n},-i s_{n}\right), 0=s_{0} \leqq s_{1} \ldots \leqq s_{n+1}=2 \pi R$, be the corresponding infinite volume limit Schwinger functions. By (3.6) they are expressed as the momenta of a generalized gaussian process with covariance $G\left(\varphi, \varphi^{\prime} ; t-t^{\prime}\right)$ which is invariant under orthogonal transformations of the sphere. Hence they are all invariant under the same transformations.

$$
\text { Let } \begin{aligned}
\eta_{1}^{i} & =R \sin \frac{\varphi^{i}}{R} & & i=0 \ldots n \\
\eta_{2}^{i} & =R \cos \frac{s^{i}}{R} \cos \frac{\varphi^{i}}{R} & & 0 \leqq s^{i} \leqq 2 \pi R \\
\eta_{3}^{i} & =R \sin \frac{s^{i}}{R} \cos \frac{\varphi^{i}}{R} & & -\frac{\pi}{2} R \leqq \varphi^{i} \leqq \frac{\pi}{2} R
\end{aligned}
$$

be a cartesian coordinatization of the sphere of radius $R$.

By the euclidean covariance:

$$
\left(\eta_{\alpha}^{i} \frac{\partial}{\partial \eta_{\beta}^{i}}-\eta_{\beta}^{i} \frac{\partial}{\partial \eta_{\alpha}^{i}}\right) S^{0}\left(f_{0}, s_{0} \ldots f_{n} s_{n}\right)=0
$$


Because the $S^{0}$ are analytic for $0=R_{e} s_{0}<R_{e} s_{1}<\ldots R_{e} s_{n}<2 \pi R$ and the $\eta$ 's are entire functions of $s_{i}$, the last equality (3.8) remains true for complex $s_{k}, s_{k}=\varepsilon_{k}+i t_{k}$

$$
0=\varepsilon_{0}<\varepsilon_{1} \ldots<\varepsilon_{n}<2 \pi R \text {. }
$$

Taking now the limit $\varepsilon_{k} \rightarrow 0,0=\varepsilon_{0}<\varepsilon_{1} \ldots<\varepsilon_{n}$ and noting that $\eta_{1} \rightarrow \xi_{1}, \eta_{2} \rightarrow \xi_{2}$, $\eta_{3} \rightarrow \xi_{3}^{\prime}$ we get the invariance of the Wightman functions under the De Sitter group.

Until now we have obtained a sequence of distributions $W_{n}^{0}\left(x_{1} \ldots x_{n}\right)$ associated with the observer in the origin, well defined if $x_{1} \ldots x_{n}$ are in the visible universe of this observer and invariant under De Sitter transformations that do not take the points out of the visible universe of the observer in the origin. But because of the symmetry of the De Sitter universe under rotations around $\xi_{3}$ axis, we can associate with any other observer, displaced from the origin by a rotation $\theta$, a set of Wightman functions defined by

$$
W_{n, \theta}^{0}\left(\theta x_{1} \ldots \theta x_{n}\right)=W_{n}^{0}\left(x_{1} \ldots x_{n}\right)
$$

that is

$$
W_{n, \theta}^{0}\left(x_{1} \ldots x_{n}\right)=W_{n}^{0}\left(\theta^{-1} x_{1} \ldots \theta^{-1} x_{n}\right) .
$$

This definition is perfectly consistent and, as a matter of fact, independent of the observer. In fact, if the points $x_{1} \ldots x_{n}$ are in the intersection of the visible universes of the two observers (that is necessary to compare experimental results), by the invariance property

$$
W_{n}^{0}\left(\theta^{-1} x_{1} \ldots \theta^{-1} x_{n}\right)=W_{n}^{0}\left(x_{1} \ldots x_{n}\right)
$$

we get

$$
W_{n, \theta}^{0}\left(x_{1} \ldots x_{n}\right)=W_{n}^{0}\left(x_{1} \ldots x_{n}\right) .
$$

So actually we have got a sequence of general Wightman functions $W_{n}^{0}\left(x_{1} \ldots x_{n}\right)$ that are well defined as long as there exists some observer s.t. $x_{1} \ldots x_{n}$ are in his visible universe and that are invariant under every transformation of the De Sitter group.

\section{The Gibbs State in the Interacting Case}

In two space-time dimensions, following the Markoff approach, we have only to construct the suitable multiplicative functional on the sphere of radius $R$, such to guarantee the invariance of the infinite volume limit Gibbs state.

Let $H_{I}^{0}$ be the free Hamiltonian and let us put $a=-b$ so that $I$ is symmetric w.r.t. the origin.

Let us choose $l \in \mathbb{R}$ such that $0<l<b$. Let us define the interacting Hamiltonian

$$
H_{I}^{l}=H_{I}^{0}+\int_{-l}^{l}: V(\phi(x)):\left(\cosh \frac{x}{R}\right)^{-2} d x,
$$

where $V(s)$ is, as usual, either a bounded below polynomial or an exponential function of the type

$$
V(s)=\int e^{\alpha s} d \mu(\alpha),
$$


where $d \mu(\alpha)$ is a positive measure with compact support contained in the interval $(-2 \pi, 2 \pi)$.

The definition of the interacting term in (4.1)

$$
\int_{-l}^{l}: V(\phi(x)):\left(\cosh \frac{x}{R}\right)^{-2} d x
$$

requires some care. The reason is that the covariance operator for the free vacuum is given by $\left(-\frac{\partial^{2}}{\partial x^{2}}+m^{2}\left(\cosh \frac{x}{R}\right)^{-2}\right)^{-\frac{1}{2}}$ and since we want to define the Wick ordering: $V(\phi(x))$ : independent of any space cutoff we have the problem that the function $\left(\cosh \frac{x}{R}\right)^{-2}$ is not bounded below by any positive constant. In fact the Wick ordering with respect to the free vacuum leads to infrared divergences of the same type as for the corresponding mass zero model in the flat case.

We shall therefore define the Wick ordering in (4.1) to be the Wick odering with respect to the free Gibbs state at temperature $T=\frac{1}{\beta}$ with $\beta=2 \pi R$. We have already seen that this Gibbs state restricted to functions of the time zero fields is actually the restriction of the Gaussian generalized process on the sphere of radius $R$ with covariance function equal to the kernel of $\left(-\Delta_{s}+m^{2}\right)^{-1}$ as a selfadjoint operator on $L^{2}(d \omega)$ where $d \omega$ is the invariant measure on the sphere and $-\Delta_{s}$ is the invariant laplacian on the sphere. By exhibiting the singularity of the kernel of $\left(-\Delta_{s}+m^{2}\right)^{-1}$ which is actually of the form

$$
\frac{1}{2 \pi} \log \sin \frac{d\left(\omega_{1}, \omega_{2}\right)}{2}
$$

where $d\left(\omega_{1}, \omega_{2}\right)$ is the geodesic distance on the sphere between the points $\omega_{1}$ and $\omega_{2}$, we immediately get that (4.2) is a well defined function of the generalized process on the sphere which is actually measurable with respect to the subalgebra generated by the fields at time zero and between $-l$ and $l$.

$H_{I}^{l}$ is essentially selfadjoint and bounded below. Moreover, as $I$ is finite, we also have that $H_{I}^{l}$ has discrete spectrum (bounded below) and $e^{-\beta H_{I} l}$ is of trace class. The computation of the trace $\operatorname{tr} e^{-\beta H_{I^{l}}}$ gives for $\beta=2 \pi R$

$$
\begin{aligned}
\operatorname{tr} e^{-2 \pi R H_{I}^{l}} & =\mid 1-e^{-\left.2 \pi R A_{I}\right|^{-1}} E_{I}^{0}\left(e^{-\int_{0}^{2 \pi R} \int_{-1}^{l}: V(\Phi(x, t)):\left(\cosh \frac{x}{R}\right)^{-2} d x d t}\right) \\
& =\left|1-e^{-2 \pi R A_{I}}\right|^{-1} E_{I}^{0}\left(e^{-\int_{0}^{2 \pi R}{ }_{-\frac{\pi}{2} R+\varepsilon}^{\frac{\pi}{2} R-\varepsilon}: V\left(\Phi^{\prime}(\varphi, t)\right): \cosh _{R} \frac{\varphi}{R} d \varphi d t}\right),
\end{aligned}
$$

where $\left[-\frac{\pi}{2} R+\varepsilon, \frac{\pi}{2} R-\varepsilon\right](\varepsilon>0)$ is the transformed by unitary operator $U^{-1}$ of the interval $[-l, l] . \Phi^{\prime}(\varphi, t)$ is the generalized gaussian process with covariance given by the Green function of the laplacian on the cut sphere and $E_{I}^{0}$ is the associated expectation.

Let us define for every $B \in B\left(L^{2}\left(d \mu_{h_{I}}\right)\right)$

$$
\alpha_{I}^{l}[t](B)=e^{-i t H_{I}^{l}} B e^{i t H_{I}^{l}}
$$

and

$$
\omega_{I}^{l}(B)=\left(\operatorname{tr} e^{-2 \pi R H_{I}^{l}}\right)^{-1} \operatorname{tr}\left(B e^{-2 \pi R H_{I}^{l}}\right) .
$$


As in the free case, for $B \in \overline{\mathscr{A}}(\Omega)$ and $\Omega_{t} \subset[-l, l] \subset I \alpha_{I}^{l}[t]$ is independent of $I$ and $l$ and gives a group of $C^{*}$-automorphism in $\overline{\mathscr{A}}$. We call $\alpha_{t}$ this independent value. We now state the main result.

Theorem 4.1. Let $\mathscr{A}$ be the local algebra for the interacting field. There exists a state $\omega$ invariant under $\alpha_{t}$

$$
\omega\left(B \cdot \alpha_{t}(C)\right)=\omega\left(\alpha_{-t}(B) \cdot C\right)
$$

for any $B$ and $C$ in $\mathscr{A} . \omega\left(B \cdot \alpha_{t}(C)\right)$ is analytic in the strip $-2 \pi R<\operatorname{Im} t<0$ and bounded and continuous in $-2 \pi R \leqq \operatorname{Im} t \leqq 0$, and satisfies the KMS condition on the boundary

$$
\omega\left(B \cdot \alpha_{t-i 2 \pi R}(C)\right)=\omega\left(C \cdot \alpha_{-t}(B)\right)
$$

for real $t$.

Furthermore if $B$ is in the subalgebra generated by operators of the form $\alpha_{t}(F)$ where $t$ belongs to a fixed finite interval and $F \in \mathscr{F}(\Omega)$ for a fixed bounded $\Omega$ then

$$
\omega(B)=\lim _{l \rightarrow \infty} \lim _{I \rightarrow \mathbb{R}} \omega_{I}^{l}(B) .
$$

Moreover $\omega$ is also invariant under the induced action of the De Sitter group on $\mathscr{A}$.

This theorem is proved in the same way as the corresponding theorem in the flat case [1]. The convergence $I \rightarrow \mathbb{R}$ goes exactly as in the free case and the convergence $l \rightarrow \infty$ is actually trivial due to the cutoff function $\left(\cosh \frac{x}{R}\right)^{-2}$ in (4.2). By what we have said above we also got the following theorem

Theorem 4.2. The Wightman functions $W_{n}\left(x_{1} \ldots x_{n}\right)$ for the interacting scalar field on the De Sitter universe of radius $R$ in two space-time dimensions are well defined for all the events $x_{1} \ldots x_{n}$ in the De Sitter universe that are simultaneously within the visible universe of some observer. $W_{n}\left(x_{1} \ldots x_{n}\right)$ are invariant under the De Sitter group and with $x_{i}=\left(\varphi_{i}, t_{i}\right)$ they are analytic in the hyperstrip $0>\operatorname{Im} t_{1} \ldots>$ $\operatorname{Im} t_{n}>-2 \pi R$.

Let the Schwinger function $S_{n}\left(\omega_{1} \ldots \omega_{n}\right)$ be the values of $W_{n}$ at the imaginary time axis, then $S_{n}\left(\omega_{1} \ldots \omega_{n}\right)$ is defined for any $\omega_{1} \ldots \omega_{n}$ on the sphere of radius $R$ and $S_{n}\left(\omega_{1} \ldots \omega_{n}\right)$ is actually invariant under rotations of this sphere. Moreover

$$
\begin{aligned}
& \int S_{n}\left(\omega_{1} \ldots \omega_{n}\right) f_{1}\left(\omega_{1}\right) \ldots f_{n}\left(\omega_{n}\right) d \omega_{1} \ldots d \omega_{n} \\
& =\frac{E^{0}\left[\prod_{j=1}^{h} \Phi\left(f_{j}\right) e^{-\int: V(\Phi(\omega)): d \omega}\right]}{E^{0}\left[e^{-\int: V(\Phi(\omega)): d \omega}\right]}
\end{aligned}
$$

where the expectation is taken w.r.t. the generalized Gaussian process $\Phi(\omega)$ with mean zero and covariance given by the kernel in $L^{2}(d \omega)$ of $\left(-\Delta_{s}+m^{2}\right)^{-1} .-\Delta_{s}$ is the invariant laplacian on the sphere, $d \omega$ is the invariant measure on the sphere and $: V(\Phi(\omega))$ : is the Wick ordering with respect to this gaussian process on the sphere.

Acknowledgements. The first and the last named authors would like to thank the members of the Mathematics Department at Oslo University for their warm hospitality. Especially we all would like to thank Dr. Sergio Albeverio for many illuminating discussions. 


\section{References}

1. Høegh-Krohn,R.: Relativistic quantum statistical mechanics in two-dimensional space-time. Commun. Math. Phys. 38, 195-224 (1974)

2. Feldman, J.: The $\lambda \varphi_{3}^{4}$ field theory in a finite volume. Commun. math. Phys. 37, 93-120 (1974)

3. Møller, C.: The theory of relativity. Oxford Clarendon Press 1960

4. Robinson, D. W.: The thermodynamic pressure in quantum statistical mechanics. Berlin Heidelberg New York: Springer 1971

5. Segal,I.: Tensor algebras over Hilbert spaces. Trans. Amer. Math. Soc. 81, 106-134 (1956)

6. Glimm, J., Jaffe, A.: Boson quantum field models. In: Streater, R. (Ed.): Mathematics of contemporary physics. New York: Academic Press 1972

7. Nelson, E.: Construction of quantum fields from Markoff fields. J. Funct. Anal. 12, 97-112 (1973)

Communicated by A. S. Wightman 